\title{
A New Model Predictive Control for the Artificial Pancreas
}

\author{
Mauro Capocelli1 ${ }^{1}$ Luca De Santis ${ }^{2}$, Vincenzo Piemonte ${ }^{2 *}$ \\ 1 Unit of Process Engineering, Department of Engineering, Università Campus Bio-Medico di Roma, via \\ Álvaro del Portillo 21, 00128 Rome, Italy. \\ 2 Unit of Chemico-physical fundamentals in Chemical Engineering, Università Campus Bio-Medico di Roma, \\ via Álvaro del Portillo 21, 00128 Rome, Italy.
}

\begin{abstract}
* Corresponding author. Tel.: 393205353406; email: v.piemonte@unicampus.it Manuscript submitted November 30, 2017; accepted January 4, 2018.

doi: 10.17706/ijbbb.2018.8.4.195-201
\end{abstract}

\begin{abstract}
Closed-loop insulin delivery system have led to significant improvement in the quality of life of subject with diabetes and are challenging to overcome the barrier of hypoglycemia, the most frequent complication of insulin therapy. The reliability of the system, composed by a computer algorithm, a glucose sensor and an insulin infusion device, depends on the knowledge and predictor capacity of the physiology of blood glucose regulation. This paper describes the physical-mathematical fundamentals and the most important results of a new three-compartmental model. The model includes exogenous insulin injected in subcutaneous tissue with local degradation, three explicit delays and three influencing physiologically-based parameters controlling the regulatory system. The parameters have been calculated through the simulation of actual clinical data and, therefore, implemented into the mathematical model to successfully simulate the clinical data obtained at Campus Biomedico in the normal-life regulation (1 day and 4 days) of diabetic patients. The estimated model parameters were physiological meaningful and provided insights on the subject's dysfunction.
\end{abstract}

Key words: Artificial pancreas, closed loop control, predictive control model, compartimental model.

\section{Introduction}

Although many innovations have been made in the field of insulin manufacturing and delivery, many patients remain unable to achieve glycemic targets and HbA1c good levels [1]. Diabetes patients suffer from high morbidity and mortality rates due to complications that could be prevented with intensive treatment and novel technologies. Over 400 million adults currently have diabetes, and this number is expected to increase by $50 \%$ in the next 25 years; The global prevalence of diabetes is estimated to be around the $10 \%$ in both males and in females [2].

Nowadays, the Artificial Pancreas (AP), investigated and discussed since the 1970s [1]-[4], has become a hot area of translational research and industrial development and likely the most promising technological development in the treatment of diabetes [3]-[6]. Artificial pancreas, schematically described in Fig. 1, consists of a closed-loop control realized through asubcutaneous system combining a sensor for the CGM, a control algorithm and an insulin infusion device.

The mathematical algorithm, included in the closed-loop control, to maintain glucose concentration within the normal range, must cope with [4]:

- complex biological mechanism in glucose-insulin control regulations resulting in non-linear 
dynamics,

- delays and inaccuracies in both glucose sensing and insulin delivery device as the intrinsic delay between the insulin action (time needed for the occurrence of the insulin absorption peak after the injection)and the measuring of the interstitial glucose concentration,

- disturbances occurring in the system due to the meals ingestions or physical activities; the AP controller has to find a trade-off between slow-pace regulation (quasi-steady state) and drastic postprandial regulation; a slow response cannot provide a good attenuation of postprandial glucose peaks and an excessive responsiveness may result in system oscillations;

The first generation of AP are actually "hybrid closed-loop systems," due to the requirement of external intervention (usually for considering the mealtime and exercise). Research efforts are still needed to delivery fully closed-loop systems, employing artificial intelligence. The class of model-predictive-control (MPC), using quantitative mathematical model of the metabolic system with delays and constrains, is the most suitable approach for the control system implementation and has overcome the difficulties encountered by the standard proportional integral derivative (PID) controllers [7], [8].

Since the performance of the control strategy depends on the model accuracy, the modeling development and validation is the most critical step in the AP improvement. Starting from the "minimal" models to the phenomenological models (based on the actual pharmacokinetics-pharmacodynamics modeling)have been proposed and validated in the literature with varying degrees of complexity, different compromises between the number of equations and the model accuracy [9]-[14]. Most of them can be characterized by the expedient of assuming a large collection of tissues and organs as compartment to simplify the phenomenology with a system of ordinary differential equations [9]-[13].

In this paper, we present the results of our MPC consisting in aglucose-insulin interactions DDEs (delay differential equations) tested on actual clinical data. The model describes the glucose-insulin interactions in three compartments: the subcutaneous (SC) compartment, the plasma compartment (including the rapidly equilibrating tissues) and the interstitial fluid (ISF) compartment (including the slowly equilibrating tissues). The DDEs includes six identifiable parameters able to account for the inter-patient variability. The availability of realistic individual models is the basis for conducting an in-silico trial: the closed-loop control can be tuned individually and then tested on each virtual patient.

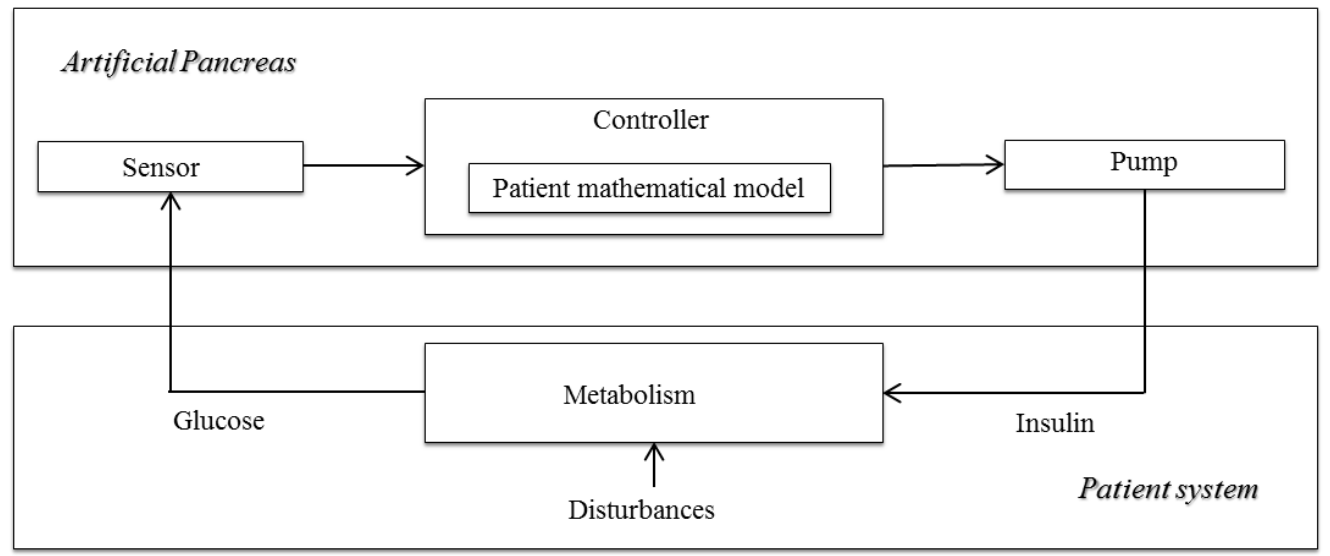

Fig. 1. Descriptive scheme of the closed chain system for glucose-insulin control in an artificial pancreas.

\section{Mathematical Modelling}

Other authors recently that a three-compartment model incorporating a ("a priori") knowledge of physiological parameters to achieve inter-patient specificity is required to describe basal glucose kinetics 
[15], [16]. In this paper we tested a three compartmental model that takes into account the main physiological factors playing a role on the blood glucose curve over time and the variability between patients (due to gender, age, weight, lifestyle, and physiological factors). The model, extending the former work of $\mathrm{Wu}$ at al. [14], includes the dynamics of the insulin releasing, the subcutaneous adsorption. And the delay caused by the absorption by sub-skin tissues and by the subcutaneous glucose sensor. There are two glucose sources (the hepatic glucose production from the liver and the glucose intake from gut) and three mechanisms of glucose utilization in the model: insulin-independent, insulin-depended and renal excretion. There are two sources of insulin in the system: the endogenous insulin secreted by the $\beta$-cells of the pancreas and the exogenous insulin injected in the subcutaneous tissue. The three compartments represented are:

1) the plasma and rapidly equilibrating tissues,

2) the ISF compartment, representing the tissues slowly equilibrating with plasma

3) the SC compartment simulating the exogenous insulin dynamic in subcutaneous tissue.

The mathematical formulation of the glucose-insulin regulatory system consists of the DDEs that follow:

$$
\begin{gathered}
\dot{G}_{P}(t)=G_{i n}(t)+H G P\left(I_{p}\left(t-\tau_{1}\right)\right)-U_{i i}\left(G_{p}(t)\right)-E\left(G_{p}(t)\right)-k_{1} G_{p}(t)+k_{2} G_{i}(t) \\
\dot{G}_{i}(t)=k_{1} G_{p}(t)-k_{2} G_{i}(t)-U_{i i}\left(G_{p}(t), I_{i}(t)\right) \\
\dot{I}_{P}(t)=k_{a 1} I_{s c}(t)+\alpha \cdot S\left(G_{p}\left(t-\tau_{2}\right)\right)-m_{1} I_{p}(t)+m_{2} I_{i}(t)-k_{e} I_{p}(t) \\
\dot{I}_{i}(t)=I_{p}(t)-m_{2} I_{i}(t)-m_{4} I_{i}(t) \\
\dot{I}_{s c}(t)=U\left(t-\tau_{3}\right)-k_{a 1} I_{s c}(t)-k_{e 1} I_{s c}(t)
\end{gathered}
$$

where $I_{p}$ and $I_{i}$ are the plasma and ISF insulin in the insulin sub-system, $I_{s c}$ is the insulin injected through the SC-compartment; $G_{p}$ is the plasma glucose and $G_{i}$ is the ISF glucose. $S$ is the insulin secreted by endocrine pancreas; HGP stands for the glucose hepatic production; $U_{i i}$ is the insulin-independent glucose utilization; $U_{i d}$ is the insulin-dependent glucose utilization; $E$ the renal excretion; $G_{i n}$ is the glucose intake rate and $U$ stands for the exogenous insulin [14], [17]. Insulin-dependent glucose utilization, depending on both insulin and glucose levels according is reported in the following equation:

$$
U_{i d}\left(G_{i}, I_{i}\right)=\beta \cdot 0.01 G_{i} / V_{g i} \cdot\left\{4+90 /\left[1+\exp \left(-1.772 \log \left[I_{i} \cdot\left(\frac{1}{V_{i i}}+\frac{0.03}{e}\right)\right]+7.76\right)\right]\right\}
$$

Insulin-independent glucose utilization is mathematically formulated by Eq 7.

$$
U_{i i}\left(G_{p}\right)=72\left[1-\exp \left(-\frac{G_{p}}{144 V_{g p}}\right)\right]
$$

The hepatic glucose production increases with the decreasing of plasma insulin concentration; the glucagon concentration is not included in this model. The function HGP, represents the effect of insulin on the glucose production. High insulin concentrations completely inhibit glucose production, whereas lower insulin concentration increases the glucose output as reported by the following equation: 


$$
H G P\left(I_{p}\right)=170 /\left[1+\exp \left(0.29\left(\frac{I_{p}}{V_{i p}}-17.5\right)\right)\right]
$$

Renal excretion occurs in the regulatory system when the plasma glucose level is higher than a threshold value as in Eq.9. The parameter values can be found in the cited literature [14], [17].

$$
E\left(G_{p}\right)=0.0005\left[G_{p}(t)-k_{e 2} \cdot B W\right]
$$

The Gut absorption profiles simulate the condition of the m-th meal ingestion $G_{m}$ at time $t_{m}$. Glucose input $G_{i n}$ is obtained by integrating all meal intake $G_{m}$ andinterpolating the experimental data.

$$
G_{i n}(t)=\sum_{\forall m \in M} G_{m}\left(t-t_{m}\right) \cdot \mathcal{U}\left(t-t_{m}\right)
$$

The model includes insulin Lispro kinetics with bolus and continuous subcutaneous insulin infusion (CSII) ways of delivery. The insulin is absorbed in subcutaneous tissue with a time delay before its appearance in plasma. Insulin secretion in the bloodstream is stimulated by high plasma glucose concentration as showed in the following equation:

$$
S\left(G_{p}\right)=210 /\left[1+\exp \left(5.21-0.003 G_{p} / V_{g p}\right)\right]
$$

Table 1 reports the definition and explanation of the inter-patient adjustable parameters reported in the

\begin{tabular}{|c|c|}
\hline$\tau_{1}$ & $\begin{array}{l}\text { Time delay of hepatic glucose production from glycogen stores (in minutes). It presented strong inter-patients } \\
\text { variability due to the complexity of the hepatic mechanisms since it is due to the liver stimulation by the plasma } \\
\text { insulin. }\end{array}$ \\
\hline$\tau_{2}$ & duction. \\
\hline$\tau_{3}$ & $\begin{array}{l}\text { Time delay of insulin subcutaneous delivery (with a little inter-patient variability due to the subcutaneous tissue } \\
\text { resistance after a prolonged use). }\end{array}$ \\
\hline $\boldsymbol{k}_{a 1}$ & $\begin{array}{l}\text { Mass transfer rate constantof exogenous insulin from subcutaneous tissue to plasma compartment; individual variable } \\
\text { parameter based on subject's clinical data (in the range } 0-1 \min ^{-1} \text { ) }\end{array}$ \\
\hline$\alpha$ & Residual capacity of pancreas to produce insulin in relation to high blood glucose level (in the range $0-1$ ). \\
\hline $\boldsymbol{\beta}$ & 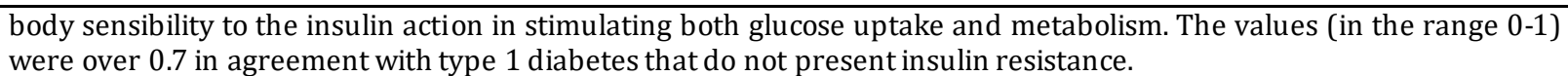 \\
\hline
\end{tabular}
model. Further details on the discussion of physiologically-based parameters are reported in the literature [14], [17].

Table 1. Definition and Explanation of the Inter-Patient Adjustable Parameter

\section{Results and Discussion}

This model has been tested by simulating the clinical data, during normal life 1 and 4 days, from T1D patients attending the Endocrinology and Diabetes Department of University Campus Bio-Medico in Rome. The therapy pump allows setting a basal rate insulin delivered all throughout the day and night for normal body function without food and a bolus (or dose insulin on demand). Some model parameters assumed fixed values for each case study, both fixed and experimentally determined through the simulation of the clinical case studies).

The identification of the six parameter of Table 1 (influencing the behaviour of glucose regulation system) allowed to tailor the simulation on the real clinical scenario (patient customization).Total time delay 
resulted in an overall $100 \mathrm{~min}$ or more; the time-to- peak of the plasma insulin concentration for short-acting insulin is 50-60 min followed by the delay in the insulin action (30-40 min) and the delay relative to the glucose diffusion from the plasma to the interstitial fluid of 10-20 min.

Fig. 2 and 3 depict the values of $G_{i}(t)$, both simulated and experimentally evaluated, relative to one 3 cases studies, the first two, reported in Fig.2, for 1 life day and the last one, reported in Fig. 3, for 4 life days.The model output is represented by a continuous blue line, the red line represents the clinical data collected by the continuous glucose monitoring sensor; at the bottom of Fig. 2 and 3, the green solid curve is the injected insulin whereas the grey dashes curve represents the gut-absorption dynamic. While insulin is represented by a discrete input with respect to time (histogram) the contribution of cairbodrati is delayed over time thanks to the use of the absorption model (a.11).
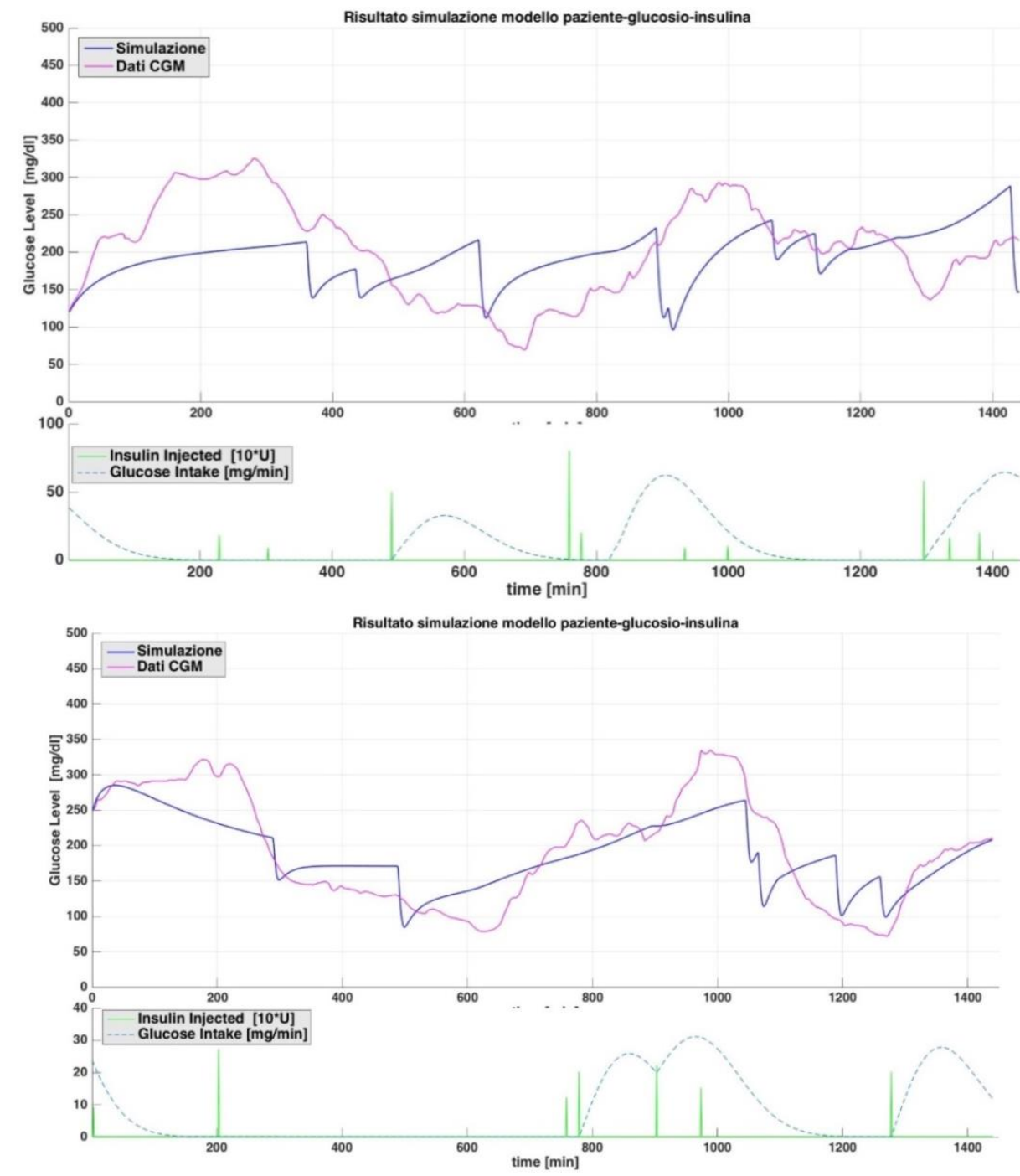

Fig. 2. Glucose level profiles with the simulation and the insulin and Glucose Intake for two patients during 1 life day.

From the aforementioned figures it is evident the good agreement, from a qualitatively point of view, between clinical data e simulated curves. Globally, the proposed model gives a good representation of biological regulation system. From the knowledge of the physiologically based parameters of Table 1, the meal glucose uptake and insulin injected (the main input of the insulin-glucose regulation model) the simulated glucose level is in good agreement with the measured pattern that significantly decreases after 
insulin injection. This model has been preliminary compared to the model of Wu et al., [14], showing promising results [17].

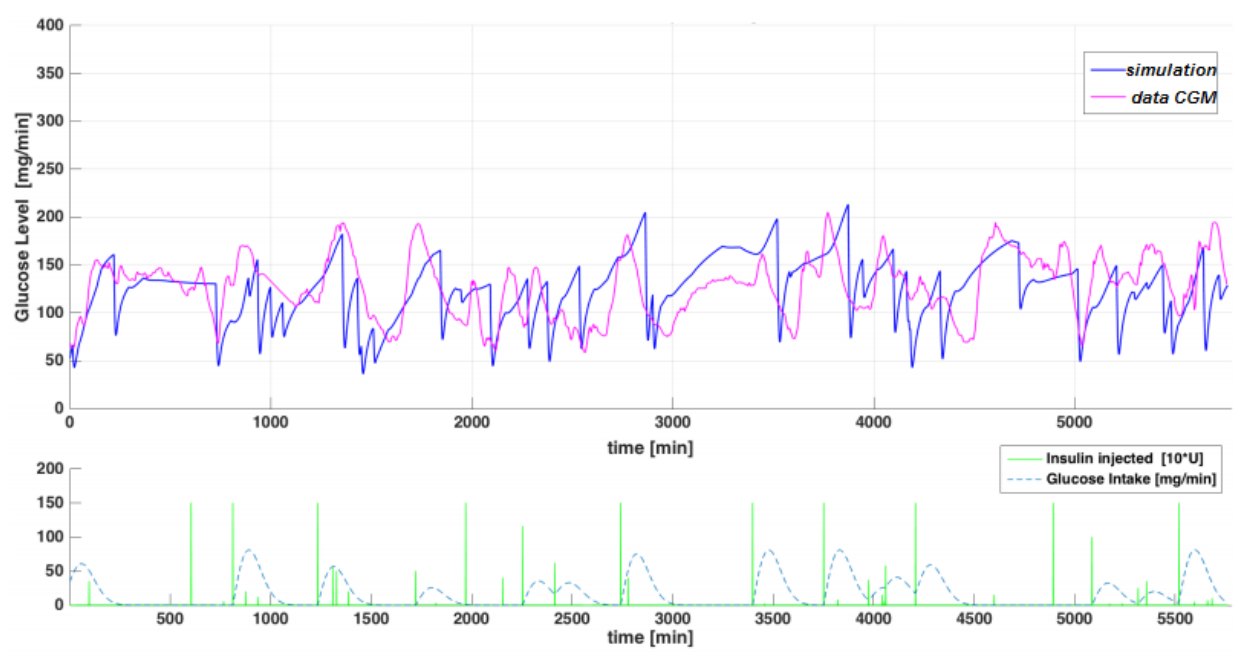

Fig. 3. Glucose level profiles with the simulation and the insulin and Glucose Intake for one patient during 4 life days.

\section{Conclusions}

MPC reached a good representation of biological regulation system as proved by the pertinent literature and the three compartmental model, here described. The differences between the simulation and measurement might be causedby other disturbances:

- the physical activity: exercise increases rates of glucose uptake and rates of endogenous glucose production must increase to meet the increased metabolic demands of the muscles to prevent hypoglycemia.

- subject's condition: glucose-insulin system may be disrupted by severe diabetes. The severity of diabetes may cause the model inability to predict glucose level. Other important factors in diabetics may be obesity, the stress and all the pathologies correlated with diabetes.

- Influences of other hormones and nervous system, usually not considered in this kind of works.

The physiologically-based approach allows to personalize the AP and to increase the clinical knowledge to better define the therapy. Although this work has the advantage of obtaining characteristic parameters of disease and physiology, our research group is working on hybrid black-box approaches to further improve the predictive capacity without losing the information regarding the actual phenomenology of the AP system.

\section{References}

[1] WHO Library Cataloguing-in-Publication DataGlobal report on diabetes. (2016).World Health Organization. ISBN 9789241565257 World Health Organization.

[2] Bequette, B. W. (2005). A critical assessment of algorithms and challenges in the development of a closed-loop artificial pancreas. Diabetes Technology \&Therapeutics, 7(1), 28-47.

[3] Vos, T., Barber, R. M., Bell, B., et al. (2015). Global, regional, and national incidence, prevalence, and years lived with disability for 301 acute and chronic diseases and injuries in 188 countries, 1990-2013: A systematic analysis for the global burden of disease study 2013. The Lancet, 386(9995), 743-800.

[4] American Diabetes Association. (2013). Economic costs of diabetes in the US in 2012. Diabetes Care, 
36(4), 1033-1046.

[5] Whiting, D. R., Guariguata, L., Weil, C., \& Shaw, J. (2011). IDF diabetes atlas: Global estimates of the prevalence of diabetes for 2011 and 2030. Diabetes Research and Clinical Practice, 94(3), 311-321.

[6] Funnell, M. M., Brown, T. L., Childs, et al. (2009). National standards for diabetes self-management education. Diabetes care, 32(Supplement 1), S87-S94.

[7] Hovorka, R. (2006). Continuous glucose monitoring and closed-loop systems. Diabetic Medicine, 23(1), 1-12.

[8] Cobelli, C., Renard, E., \& Kovatchev, B. (2011). Artificial pancreas: Past, present, future. Diabetes, 60(11), 2672-2682.

[9] Bergman, R. N. (2005). Minimal model: Perspective from 2005. Hormone Research in Paediatrics, 64(Suppl. 3), 8-15.

[10] Dalla, M. C., Raimondo, D. M., Rizza, R. A., \& Cobelli, C. (2007). GIM, simulation software of meal glucose — Insulin model.J Diabetes SciTechnol, 1, 323-330.

[11] Bergman, R. N., Ider, Y. Z., Bowden, C. R., \& Cobelli, C. (1979). Quantitative estimation of insulin sensitivity. American Journal of Physiology-Endocrinology And Metabolism, 236(6), E667.

[12] Vicini, P., Caumo, A., \& Cobelli, C. (1997). The hot IVGTT two-compartment minimal model: indexes of glucose effectiveness and insulin sensitivity. American Journal of Physiology-Endocrinology And Metabolism, 273(5), E1024-E1032.

[13] Toffol o, G., \& Cobelli, C. (2003). The hot IVGTT two-compartment minimal model: An improved version. American Journal of Physiology-Endocrinology and Metabolism, 284(2), E317-E321.

[14] Wu, Z., Chui, C. K., Hong, G. S., \& Chang, S. (2011). Physiological analysis on oscillatory behavior of glucose-insulin regulation by model with delays. Journal of theoretical biology, 280(1), 1-9.

[15] Insel, P. A., Liljenquist, J. E., Tobin, J. D., Sherwin, R. S., Watkins, P., Andres, R., \& Berman, M. (1975). Insulin control of glucose metabolism in man: a new kinetic analysis. The Journal of Clinical Investigation, 55(5), 1057-1066.

[16] Cobelli, C., Toffolo, G., \& Ferrannini, E. (1984). A model of glucose kinetics and their control by insulin, compartmental and noncompartmental approaches. Mathematical biosciences, 72(2), 291-315.

[17] Piemonte, V., Capocelli, M., Santis, L., Maurizi, A. R., \& Pozzilli, P. (2017). A Novel Three-compartmental model for artificial pancreas: Development and validation. Artificial organs, 41(12), 326-336.

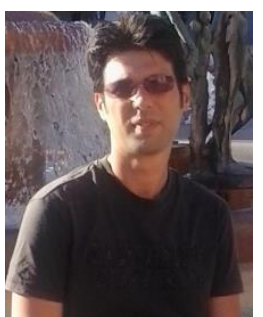

Vincenzo Piemonte is an associate professor at the University "Campus Bio-medico" of Rome (chairs on artificial organs engineering, chemical and biochemical engineering principles, biorefinery principles); He has been an adjunct professor at the Department of Chemical Engineering of the University "La Sapienza" of Rome (chair on artificial organs engineering).

His research activity is primarily focused on the study of Transport phenomena in the artificial and bioartificial organs; new biotreatment technology platform for the elimination of toxic pollutants from water and soil; Life Cycle Assessment (LCA) of petroleum-based plastics and bio-based plastics; extraction of valuable substances (polyphenols, tannins) from natural matrices; hydrogen production by membrane reactors for water gas shift reaction; concentrated Solar Power Plant integrated with membrane steam reforming reactor for the production of hydrogen and hydro-methane.

He has about 150 publications on chemical thermodynamics, kinetics, biomedical devices modelilng, Bioreactors, LCA studies. 